\title{
Inhibition of Zymosan-Induced Inflammatory Factors Expression by ATRA Nanostructured Lipid Carriers
}

\author{
Hongyan Zhou, ${ }^{1}$ Wensong Zhang, ${ }^{2}$ Xunyi Gao, ${ }^{1}$ Hongguang Zhang, ${ }^{3}$ and Ning Kong ${ }^{4}$ \\ ${ }^{1}$ Department of Ophthalmology, China-Japan Union Hospital of Jilin University, Changchun 130033, China \\ ${ }^{2}$ Department of Ophthalmology, The Second Hospital of Jilin University, Changchun 130000, China \\ ${ }^{3}$ Department of Chemical Engineering and Application, College of Chemistry, Jilin University, Changchun 130000, China \\ ${ }^{4}$ Department of Pharmacy, College of Pharmacy, Jilin University, Changchun 130041, China
}

Correspondence should be addressed to Wensong Zhang; zhangwensong2016@163.com

Received 25 January 2016; Revised 20 April 2016; Accepted 28 April 2016

Academic Editor: Taras Ardan

Copyright (c) 2016 Hongyan Zhou et al. This is an open access article distributed under the Creative Commons Attribution License, which permits unrestricted use, distribution, and reproduction in any medium, provided the original work is properly cited.

\begin{abstract}
Purpose. The study aimed to evaluate the effect of all-trans retinoic acid-loaded nanostructured lipid carriers (ATRA-NLCs) on the zymosan-induced expression of the cytokines IL-4, IL-10, and IFN- $\gamma$ and the matrix metalloproteinases/tissue inhibitor of metalloproteinases (MMPs/TIMPs) and $\mathrm{TLR}_{2}$ in rabbit corneal fibroblasts (RCFs). Methods. ATRA-NLCs were prepared by emulsification. RCFs were isolated and harvested after four to seven passages in monolayer culture. Cytokine release (IL-4, IL-10, and IFN- $\gamma$ ) induced by zymosan was analyzed by cytokine release assay, reverse transcription, and real-time polymerase chain reaction (RT-PCR) analysis detection. MMP-1, MMP-3, and MMP-13, TIMP-1 and TIMP-2, and TLR2 expression were analyzed by immunoblotting. Results. ATRA-NLCs were resistant to light and physically stable, and the average size of the ATRA-NLCs was $200 \mathrm{~nm}$. ATRA-NLCs increased the zymosan-induced release of IL- 4 and IL-10 and decreased the release of IFN- $\gamma$ by RCFs. ATRA-NLCs decreased the levels of TLR2 and MMPs/TIMPs above. Conclusions. ATRA may be a potent anti-inflammatory agent for the therapy of fungal keratitis (FK).
\end{abstract}

\section{Introduction}

Fungal keratitis (FK) is an ulcerative and sight-threatening infection of the cornea that sometimes leads to blindness. Although patients with FK have been treated with antifungal drugs that prevent the progression of corneal pathogenesis, further study is still needed. The objectives of this study were to investigate the effect of ATRA-NLC on the zymosaninduced expression of the cytokines IL-4, IL-10, IFN- $\gamma$, MMPs/TIMPs, and $\mathrm{TLR}_{2}$.

Zymosan, a $\beta$-glucan component of the yeast cell wall, is a ligand of TLR $R_{2}$ and dectin-1 which stimulates macrophages to produce proinflammatory mediators [1]. Zymosan can also regulate $\mathrm{TLR}_{2}$ gene expression in the context of neuroinflammation [2]. Fungal inflammation is mediated by the expression of $\mathrm{TLR}_{2}$ activation. TLRs may activate the innate immune system. TLRs have been used as a legitimate therapeutic strategy. Zymosan-induced $\mathrm{TLR}_{2}$ expression was investigated in this study.
MMPs are enzymes involved in leukocyte diapedesis and migration to the inflammatory focus. MMP-2, MMP-9, and TIMP-1 play specific roles in the pathogenesis of FK [3]. Specifically, the levels of MMP-8, MMP-9, MMP-13, and TIMP-1 increase during the early stages of $C$. albicans keratitis [4], and the ratio of MMPs/TIMPs is higher in the FK corneas. Corneal polymorphonuclear neutrophils (PMNs) that infiltrate the cornea of patients with FK increase the activities of MMP-8 and MMP-9, thereby enhancing tissue destruction [5]. Therefore, this study was to determine the association between the MMP/TIMP and zymosan-induced inflammation to clarify the role of MMP/TIMP in the pathology of FK and then to study the potential role of new therapeutic method.

IL- 4 and IL-10 play a role in protective immunity against infections with extracellular parasites and are responsible for inflammatory diseases. IFN- $\gamma$ is secreted by activated Th1 and other immune cells, and it creates proinflammatory microenvironments [6]. Zymosan-induced production of 
the cytokines IL-4, IL-10, and IFN- $\gamma$ by RCFs was examined in this study.

Nuclear receptors are ligand-activated transcription factors that signal the expression of the genome. Understanding the expression of receptors and their activating lipid ligands in immune diseases will be crucial for the development of new therapies to target nuclear receptors [7]. ATRA is a bioactive derivative of vitamin $\mathrm{A}$ that has demonstrated immunomodulatory effects in many immune disorders [8]. ATRA is a crucial immunostimulatory cofactor that activates macrophages and their subsequent differentiation into dendritic-like cells [9]. Moreover, ATRA exerts an antiinflammatory effect on monocytes via $\mathrm{TLR}_{2 / 1}$ and CD14 expression [10, 11]. ATRA exacerbates allergic immune and inflammatory responses, most likely by promoting Th 2 development [12]. Thus, ATRA may be a novel strategy to treat inflammation in humans, but it is poorly soluble, which hinders its ocular delivery. In this study, we designed NLCs to overcome these barriers, which led to poor bioavailability. Because ATRA exhibits anti-inflammatory activity [10], we investigated the effect of ATRA-NLCs on the zymosaninduced expression of TLR 2 , IL-4, IL-10, and IFN- $\gamma$, as well as the function of MMP/TIMP in RCFs. The potential role of ATRA-NLCs in the therapy of FK was clarified.

\section{Materials and Methods}

Dispersant, collagenase, Eagle's minimum essential medium (MEM), Dulbecco's phosphate-buffered saline (DPBS), Fetal Bovine Serum (FBS), (Sigma), ATRA (Sigma Aldrich), Whales wax palm, soybean oil (Aladdin Reagent company), lecithin (Shanghai Huishi Biochemicals company), dibutylhydroxytoluene (chemically pure, Sinopharm Group Chemical Reagent company), oleic acid (chemically pure, Recovery of Tianjin Institute of Fine Chemicals), and Poly-Yamanashi Ester, 80 (East China reagent factory in Tianjin) were used. The following antibodies were used: MMP-1 and MMP-3 and TIMP-1 (RD), MMP-13 (BioVision), TIMP-2 and TLR 2 (Boster), and GAPDH (Cell signal).

Anti-mouse IgG-HRP (Abcam) and goat anti-rabbit IgGHRP (RD) were used. Trizol Reagent (Invitrogen) LightCycler $^{\circledR} 480$ Real-Time PCR System (Roche Diagnostics GmbH) and Primer were synthesized by Sangon Biotech Co. Ltd. (Shanghai, China). Reverse transcription system (Promega) was used. The following equipment was used: a DF-101S hottype constant temperature heating magnetic stirrer (Shanghai Yukang Science and Education Equipment Co.), an Ultrasonic Cell Crusher (Ningbo Xingzhi Biotechnology Co.), a high-performance liquid chromatograph (Agilent), a ZF-I ultraviolet analyzer (Shanghai Gucun Electro-Optical Instrument company), a particle size analyzer (Brookhaven Instruments), and an electronic universal oven (Beijing everlasting light medical instrument company).

2.1. ATRA-NLC Preparation. ATRA-NLC was prepared by emulsification according to the process shown in Figure 1.

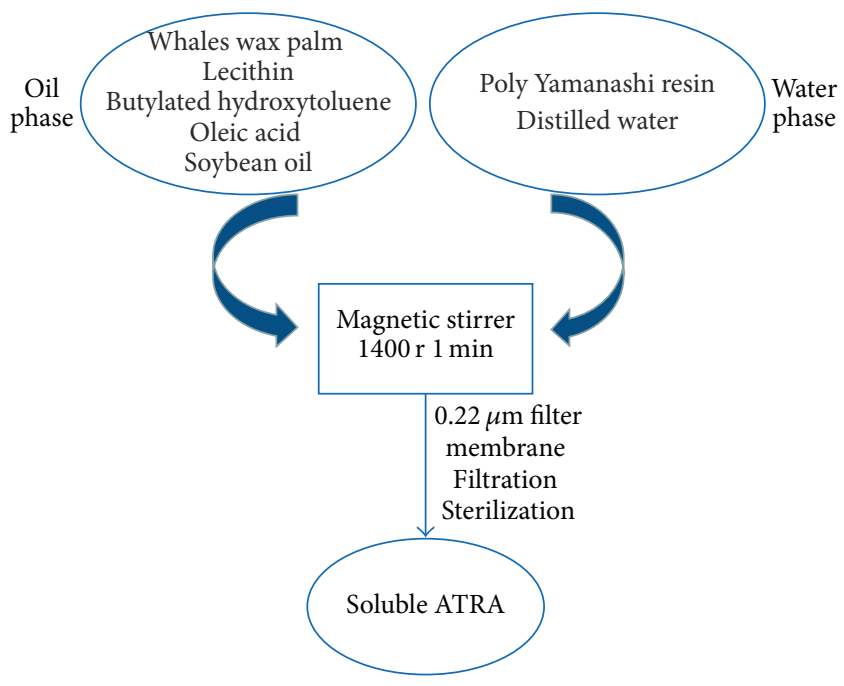

FIgURE 1: The method used to prepare ATRA-NLCs.

2.2. Cell Isolation. New England white rabbits ( 2.0 to $2.5 \mathrm{~kg}$ ) were obtained from the animal department of Jilin University. This study adhered to the ARVO Statement for the Use of Animals in Ophthalmic and Vision Research and was approved by the Animal Experimental Committee of Jilin University. RCFs were isolated as follows: briefly, the enucleated eye was washed with DPBS containing an antibiotic-antimycotic mixture; the endothelial layers and the epithelial sheets of the excised corneas were removed, and the remaining corneal tissue was cultured with collagenase $(2 \mathrm{mg} / \mathrm{mL}$, in MEM) at $37^{\circ} \mathrm{C}$. Cells were harvested after four to seven passages in monolayer culture.

2.3. Cytokine Release Assay. RCFs ( 30000 cells $/ \mathrm{mL}$ ) were cultured in 24-well plates for $24 \mathrm{~h}$ with MEM alone. Serumfree RCFs were incubated with or without ATRA-NLC in the presence or absence of zymosan for $24 \mathrm{~h}$. The IL- 4 , IL10 , and IFN- $\gamma$ concentrations were then examined with a cytokine assay. Standard proteins were dissolved in MEM to generate standard curves. Data are presented as the mean values $\pm \mathrm{SD} .{ }^{*} P<0.01$ versus the corresponding value for cells cultured with zymosan (Dunnett T3). Each cytokine assay was repeated at least three times.

2.4. Quantitative Real-Time Polymerase Chain Reaction (RTPCR) Analysis. RCFs were cultured in $60 \mathrm{~mm}$ dishes for $24 \mathrm{~h}$ with MEM alone. Serum-free RCFs were incubated with ATRA-NLCs for 1 hour and then for an additional 4 hours in the presence or absence of Zymosan. Total RNA was isolated from RCFs in $60 \mathrm{~mm}$ culture dishes with the use of Trizol Reagent and was subjected to RT. The resulting cDNA was subjected to real-time polymerase chain reaction (PCR) analysis with specific primers for IL4, IL-10, and IFN- $\gamma$ or glyceraldehyde-3-phosphate dehydrogenase (GAPDH) by the use of a LightCycler 480 RealTime PCR System. The sequences of the PCR primers were designed by the literature previously [13]: the primers for 
IL-4 were GTTTCCCTGCTTTGAGATGG (forward) and TCAGGAAACAGCTTCGGAGT (reverse), the primers for IL-10 were TTTAGGCGAGAAGCTGAAGG (forward) and TCTTCACAGGGCAGGAATCT (reverse), the primers for IFN- $\gamma$ were TGAACATGATGGATCGTTGG (forward) and CATTCACTTTGCTGGCAGTG (reverse), and the primers for GAPDH were AACTTTGGCATTGTGGAAGGA (forward) and AACATCATCCCTGCTTCCAC (reverse).

2.5. Immunoblot Analysis. Immunoblot analyses of MMP1, MMP-3, MMP-13, TIMP-1, TIMP-2, and TLR 2 were also performed as described previously [14]. In brief, cells $(5 \times$ $10^{5}$ cells per well of a 24 -well plate) were cultured for $24 \mathrm{~h}$ in MEM and then incubated for $12 \mathrm{~h}$ with or without $0.01-$ $0.1 \mu \mathrm{mol} / \mathrm{L}$ ATRA-NLCs, followed by $30 \mathrm{~min}$ with or without zymosan $(500 \mu \mathrm{g} / \mathrm{mL})$. The cell lysates $(30 \mu \mathrm{g}$ of protein) were collected, and then the cell lysates were subjected to immunoblot analyses. Data are representative of three experiments.

\section{Results and Discussion}

3.1. ATRA-NLC Evaluation. ATRA-NLCs were light-resistant and physically stable, and they exhibited an average size of $200 \mathrm{~nm}$. Precipitation and crystallization were not observed when the ATRA-NLCs were stored for one month at $4^{\circ} \mathrm{C}$. High-speed centrifugal for 2 hours also works well.

3.2. Effects of ATRA-NLC on Zymosan-Induced IL-4, IL10 , and IFN- $\gamma$ from RCFs. The effects of ATRA-NLC at various concentrations $(0,0.01$, and $0.1 \mu \mathrm{mol} / \mathrm{mL})$ on the release of IL-4, IL-10, and IFN- $\gamma$ induced by zymosan from RCFs were investigated first (Figures 2(a), 2(b), and 2(c)). Further, quantitative RT-PCR analysis revealed that ATRANLC increased the amounts of IL-4 and IL- 10 mRNAs in RCFs induced by zymosan, and the amount of IFN- $\gamma$ was inhibited in a dose-dependent manner (Figures 2(a), 2(b), 2(c), 2(d), 2(e), and 2(f)).

3.3. Effects of ATRA-NLC on MMP-1, MMP-3, and MMP-13 and TIMP-1 and TIMP-2 TLR 2 Expression in RCFs. RCFs were stimulated by zymosan. The RCFs were incubated with or without ATRA-NLCs $(0.01-0.1 \mu \mathrm{mol} / \mathrm{L})$ for $24 \mathrm{~h}$, followed by incubation with zymosan $(500 \mu \mathrm{g} / \mathrm{mL})$ for $30 \mathrm{~min}$. The immunoblot analysis showed that the levels of MMP-1, MMP3 , and MMP- 13 and TIMP- 1 and TIMP- 2 TLR $_{2}$ were increased by stimulation with zymosan, and this increase was inhibited by ATRA-NLCs (Figure 3).

\section{Discussion}

Natamycin 5\% suspension, voriconazole, and topical amphotericin-B $0.015 \%$ constitute routine antifungal therapy for FK [15]. However, existing methods cannot meet the demands of clinical treatment, and new drugs are necessary. New antifungal therapies commonly target ergosterol, an essential constituent of fungal membranes that are not reluctant to microbial resistance and are minimally toxic to mammalian cells [16]. We have shown that ATRANLCs inhibited the zymosan-induced expression of the cytokines IFN- $\gamma$, MMPs/TIMPs, and TLR 2 and increased the zymosan-induced expression of the cytokines IL- 4 and IL-10 by RCFs.

The immune dominance of Th1 (IFN- $\gamma$ and IL-2) may be characteristic of many immune-inflammation pathogeneses [17]. The Thl response was maintained after infection because IFN- $\gamma$ production is based mainly on $\mathrm{CD}^{+} \mathrm{T}$ cells [18]. Th1 cells are involved in all forms of dry eye, which is characterized by an inflammatory pathophysiology [19]. Th1 was expected to be a target in ocular anti-inflammatory therapy. Il-4 administration may inhibit the inflammatory response and the infiltration of phenotypic macrophages to suppress TNF- $\alpha$-induced osteocyte apoptosis [12]. IL4 and IL-10 can inhibit both Thl cytokine expression and the consequent inflammation. Moreover, zymosan induces the expression of pro- and anti-inflammatory factors and Tregs. Specifically, zymosan increases the levels of IL-4, IL10, and Foxp3-positive T cells [20]. ATRA can also inhibit the expression of Thl-associated (IFN- $\gamma$ ) cytokines and increase the levels of Th2-associated cytokines (IL-4, IL-5, and IL13) in PBMCAs and purified T cells. ATRA promotes the expression of Th2 cytokines rather than Th1 cytokines, as observed in many cell lines [21]. In this study, ATRA-NLCs increased zymosan-induced IL-4 and IL-10 expression and inhibited zymosan-induced IFN- $\gamma$ expression in a dosedependent manner by cytokine release assay and RT-PCR detection, which means that ATRA-NLCs can decrease fungiassociated corneal inflammation molecular expression.

Zymosan-induced MMP-9 expression at the single-cell level during peritonitis is a quantitative indicator of the phase-specific contribution of mast cells, macrophages, and neutrophils [22]. Zymosan-induced MMP-9 production in neutrophils also affects matrix remodeling [23]. This study investigated the effects of ATRA on zymosan-induced inflammation in corneal fibroblasts, including the regulation of matrix metalloproteinases (MMPs)/tissue inhibitor of metalloproteinase (TIMP). Previous studies have demonstrated that vitamin $\mathrm{A}$ and carotenoids regulate immune function in lymphocytes and splenocytes and that the carotenoid lutein regulates matrix metalloproteinase-9 (MMP-9) production in macrophages [24]. ATRA also inhibited TGF$\beta$-induced collagen gel contraction mediated by human corneal fibroblasts by attenuating the production of MMP1 and MMP-3 [25]. ATRA downregulated MMP expression to exert an antimetastatic effect, induce differentiation and apoptosis, and inhibit proliferation and inflammation [24]. ATRA may inhibit MMP expression in different diseases and act as a strong anti-inflammatory agent $[18,24]$. The data show that ATRA-NLCs can inhibit the zymosaninduced expression of MMP-1, MMP-3, and MMP-13 and TIMPs in corneal fibroblasts. This effect may be attributed to an imbalance in the expression of proteolytic enzymes and their inhibitors, as described previously [26]. In brief, TIMP-1 and TIMP-2 are produced and related to MMP1 and MMP-9 in the context of corneal injury. The ratio of MMP/TIMP controls inflammation and is an indicator of tissue damage. ATRA-NLCs can attenuate MMP/TIMP 


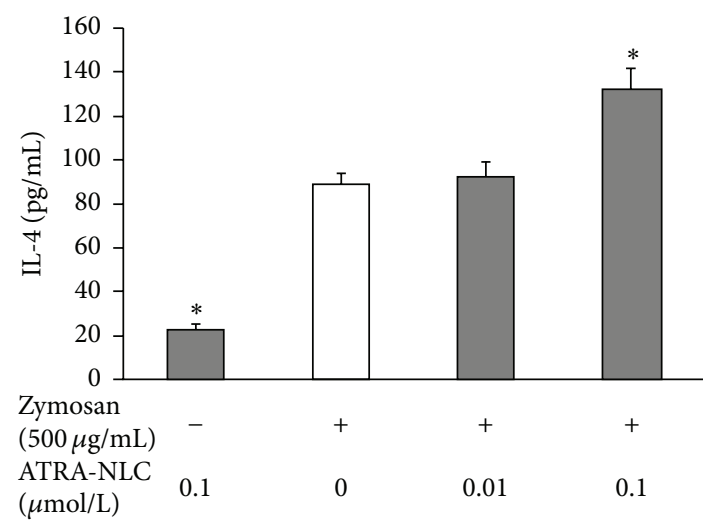

(a)

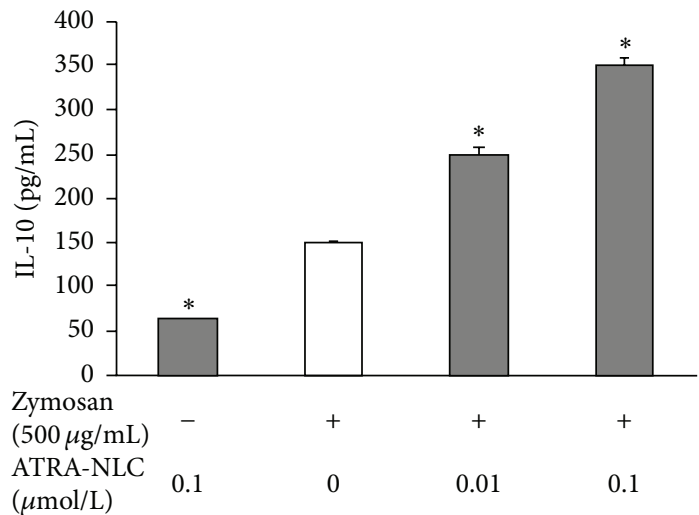

(c)

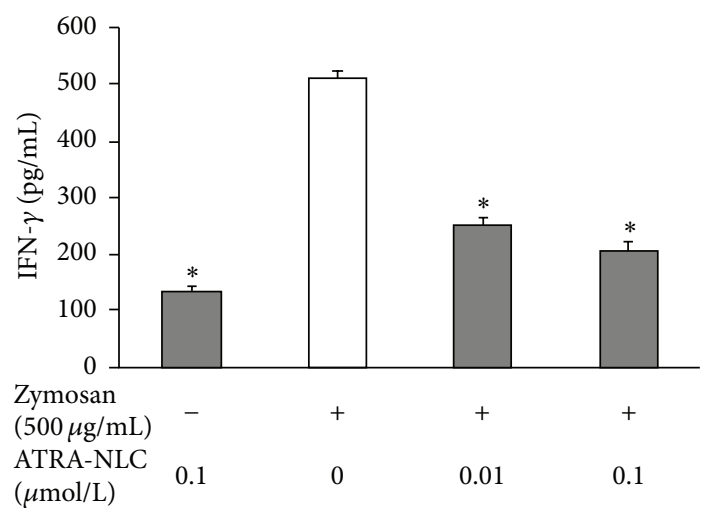

(e)

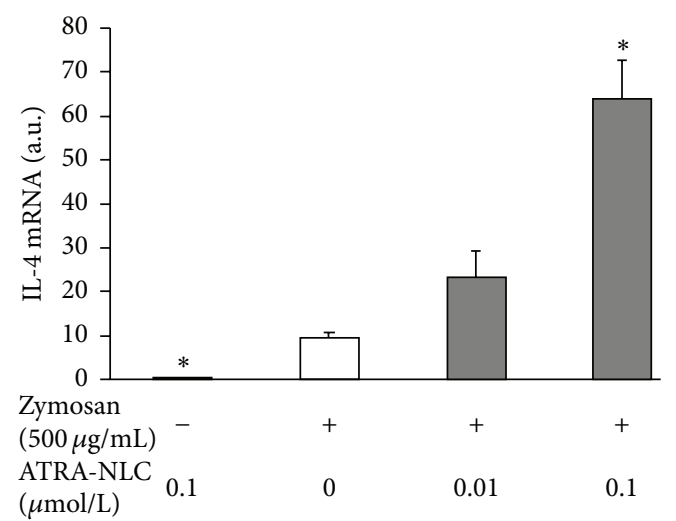

(b)

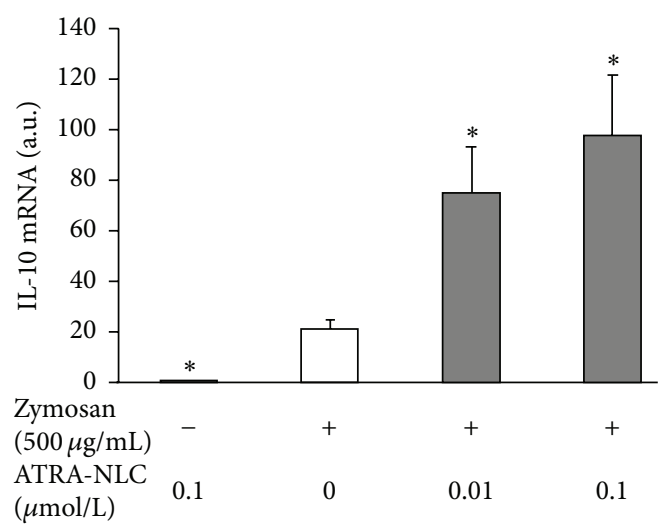

(d)

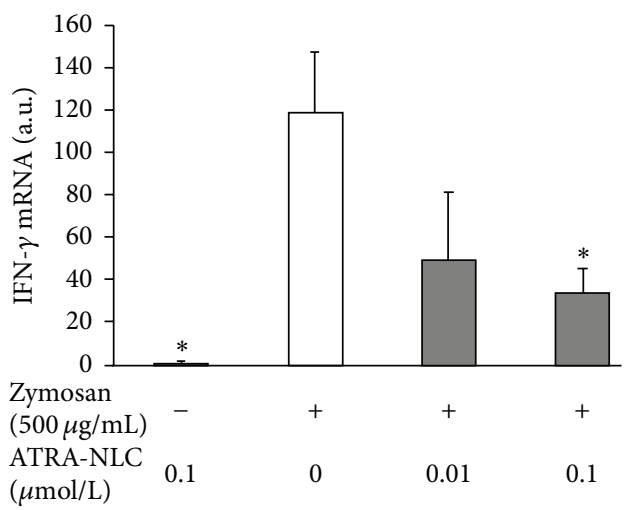

(f)

FIGURE 2: Effect of ATRA-NLC on the expression of IL-4, IL-10, and IFN- $\gamma$ from RCFs and in RCFs. (a), (c), and (e) Serum-deprived RCFs were incubated in the absence or presence of ATRA-NLC with or without zymosan for $24 \mathrm{~h}$. The IL-4, IL-10, and IFN- $\gamma$ concentrations were examined at $24 \mathrm{~h}$ with a cytokine assay system. Zymosan can induce IL-4, IL-10, and IFN- $\gamma$ release. ATRA-NLC further increased the release of IL- 4 and IL-10 and decreased the release of IFN- $\gamma$. Data are the mean values \pm SDs of three experiments. ${ }^{*} P<0.01$ versus the corresponding value for cells cultured with zymosan (Dunnett T3). (b), (d), and (f) RCFs were incubated in the absence or presence of the indicated concentrations of ATRA-NLC and zymosan, after which total RNA was isolated and subjected to RT and real-time PCR analysis of IL-4, IL-10, and IFN- $\gamma$ mRNAs. Incubation of the cells with ATRA-NLC and zymosan induced the expression of IL- 4 and IL-10 and decreased the expression of IFN- $\gamma$ in a concentration-dependent manner. Data are the mean values \pm SDs of three experiments. ${ }^{*} P<0.05$ versus the corresponding value for cells cultured with zymosan (Dunnett T3).

expression and thus could potentially inhibit FK pathology process.

$\mathrm{TLR}_{2}$, a regulatory element in the innate immunity of corneal keratocytes, rapidly and transiently induces inflammatory mediators. Antagonists of $\mathrm{TLR}_{2}$ may favor the inhibition of signaling responsible for autoimmune responses $[27,28]$. Our data show that ATRA-NLCs inhibited the production of inflammatory mediators, that is, MMPs, induced by the $\mathrm{TLR}_{2}$ ligand zymosan, and served as an inhibiting feedback regulator of $\mathrm{TLR}_{2}$ stimulation. 


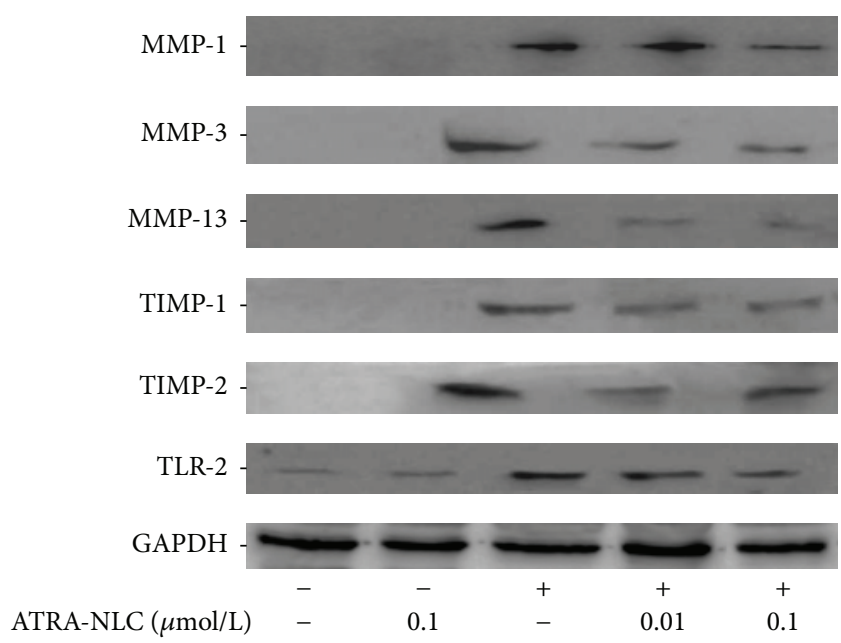

Figure 3: The effects of ATRA-NLCs on MMP-1, MMP-3, and MMP-13 and TIMP-1 and TIMP-2 TLR 2 expression in RCFs stimulated with zymosan. RCFs were incubated with or without ATRANLC for $24 \mathrm{~h}$ and then treated with zymosan. The immunoblot analysis showed that ATRA-NLCs inhibited the zymosan-mediated stimulation of MMP-1, MMP-3, and MMP-13 and TIMP-1 and TIMP-2 TLR $_{2}$.

The pharmacokinetics of potentially therapeutic peptides for the ocular surface, including the residence time of peptides at the cornea, their ability to penetrate the cornea, and the stability of the peptides, has been described [29]. Many studies have attempted to improve the efficacy of therapeutic peptides by improving the residence time. The low bioavailability and poor therapeutic response of traditional ophthalmic formulations is due to a reduced precorneal residence time of the formulation at the ocular surface [30]. NLCs can improve the oral bioavailability of poorly watersoluble drugs. Specifically, NLCs carrying drugs were more effective than the free drugs were because they increased the drug bioavailability [31]. ATRA is highly lipophilic and poorly soluble in water [26], and NLCs were able to prolong the release of ATRA [32]. ATRA-NLCs in this study showed light resistance, proper size, and good physical stability.

The results of this study suggest that ATRA negatively regulates the expression of MMPs, TIMPs, TLR 2 , and IFN- $\gamma$ and positively regulates IL- 4 and IL-10 expression. Moreover, ATRA-NLCs play major roles as anti-inflammatory agents in the context of infection via the factors described herein. Thus, ATRA may be useful as an anti-inflammatory agent in the treatment of FK.

\section{Competing Interests}

The authors declare that they have no conflict of interests.

\section{Acknowledgments}

This work was supported by Grants from the Natural Science Foundation of China (no. 81300727) and the Jilin University basic scientific research operating expenses fund (Research Fund of the Bethune B Plan of Jilin University, 2012230).

\section{References}

[1] B. S. Kim, I. S. Cho, S. Y. Park, G. Schuller-Levis, W. Levis, and E. Park, "Taurine chloramine inhibits NO and TNF- $\alpha$ production in zymosan plus interferon- $\gamma$ activated RAW 264.7 cells," Journal of Drugs in Dermatology, vol. 10, no. 6, pp. 659-665, 2011.

[2] C. Marinelli, R. Di Liddo, L. Facci et al., "Ligand engagement of Toll-like receptors regulates their expression in cortical microglia and astrocytes," Journal of Neuroinflammation, vol. 12, no. 1, article 244, 2015.

[3] S. D. Boveland, P. A. Moore, J. Mysore et al., "Immunohistochemical study of matrix metalloproteinases-2 and -9 , macrophage inflammatory protein-2 and tissue inhibitors of matrix metalloproteinases-1 and -2 in normal, purulonecrotic and fungal infected equine corneas," Veterinary Ophthalmology, vol. 13, no. 2, pp. 81-90, 2010.

[4] X. Yuan, B. M. Mitchell, and K. R. Wilhelmus, "Expression of matrix metalloproteinases during experimental Candida albicans keratitis," Investigative Ophthalmology and Visual Science, vol. 50, no. 2, pp. 737-742, 2009.

[5] G. Rohini, P. Murugeswari, N. V. Prajna, P. Lalitha, and V. Muthukkaruppan, "Matrix metalloproteinases (MMP-8, MMP9) and the tissue inhibitors of metalloproteinases (TIMP-1, TIMP-2) in patients with fungal keratitis," Cornea, vol. 26, no. 2, pp. 207-211, 2007.

[6] L. Wang, Y. Zhao, Y. Liu et al., "IFN- $\gamma$ and TNF- $\alpha$ synergistically induce mesenchymal stem cell impairment and tumorigenesis via $\mathrm{NF} \kappa \mathrm{B}$ signaling," Stem Cells, vol. 31, no. 7, pp. 1383-1395, 2013.

[7] M. Kiss, Z. Czimmerer, and L. Nagy, “The role of lipid-activated nuclear receptors in shaping macrophage and dendritic cell function: from physiology to pathology," Journal of Allergy and Clinical Immunology, vol. 132, no. 2, pp. 264-286, 2013.

[8] N. Behairi, M. Belkhelfa, H. Mesbah-Amroun et al., "Alltrans-retinoic acid modulates nitric oxide and interleukin17A production by peripheral blood mononuclear cells from patients with Alzheimer's disease," Neuroimmunomodulation, vol. 22, no. 6, pp. 385-393, 2015.

[9] H.-Y. Cho, E.-K. Choi, S.-W. Lee et al., "All-trans retinoic acid induces TLR-5 expression and cell differentiation and promotes flagellin-mediated cell functions in human THP-1 cells," Immunology Letters, vol. 136, no. 1, pp. 97-107, 2011.

[10] P. T. Liu, S. R. Krutzik, J. Kim, and R. L. Modlin, “Cutting edge: all-trans retinoic acid down-regulates TLR2 expression and function," The Journal of Immunology, vol. 174, no. 5, pp. 2467-2470, 2005.

[11] H.-L. Son, H.-R. Park, Y.-J. Park, and S.-W. Kim, "Effect of retinoic acid in a mouse model of allergic rhinitis," Allergy, Asthma and Immunology Research, vol. 7, no. 6, pp. 590-598, 2015.

[12] M. Maret, C. Ruffie, B. Periquet et al., "Liposomal retinoic acids modulate asthma manifestations in mice," Journal of Nutrition, vol. 137, no. 12, pp. 2730-2736, 2007.

[13] I. E. Maher, J. E. Griffith, Q. Lau, T. Reeves, and D. P. Higgins, "Expression profiles of the immune genes CD4, CD $8 \beta, \operatorname{IFN} \gamma$, IL-4, IL-6 and IL-10 in mitogen-stimulated koala lymphocytes (Phascolarctos cinereus) by qRT-PCR," PeerJ, vol. 2, article e280, 2014. 
[14] H.-Y. Zhou, J.-L. Hao, M.-M. Bi, S. Wang, H. Zhang, and W.S. Zhang, "Molecular mechanism of the inhibition effect of Lipoxin A4 on corneal dissolving pathology process," International Journal of Ophthalmology, vol. 6, no. 1, pp. 39-43, 2013.

[15] S. Solanki, M. Rathi, S. Khanduja, C. S. Dhull, S. Sachdeva, and J. Phogat, "Recent trends: medical management of infectious keratitis," Oman Journal of Ophthalmology, vol. 8, no. 2, pp. 8385, 2015.

[16] J. F. Aparicio, E. G. Barreales, T. D. Payero, C. M. Vicente, A. de Pedro, and J. Santos-Aberturas, "Biotechnological production and application of the antibiotic pimaricin: biosynthesis and its regulation," Applied Microbiology and Biotechnology, vol. 100, no. 1, pp. 61-78, 2016.

[17] J. F. Meadows, K. Dionne, and K. K. Nichols, "Differential profiling of T-cell cytokines as measured by protein microarray across dry eye subgroups," Cornea, vol. 35, no. 3, pp. 329-335, 2016.

[18] L. Qin, W. Xu, X. Li et al., "Differential expression profile of immunological cytokines in local ovary in patients with polycystic ovarian syndrome: analysis by flow cytometry," European Journal of Obstetrics \& Gynecology and Reproductive Biology, vol. 197, pp. 136-141, 2016.

[19] X. Wu, X. Feng, Y. He et al., "IL-4 administration exerts preventive effects via suppression of underlying inflammation and TNF- $\alpha$-induced apoptosis in steroid-induced osteonecrosis," Osteoporosis International, vol. 27, no. 5, pp. 1827-1837, 2016.

[20] J. Zhu, "T helper 2 (Th2) cell differentiation, type 2 innate lymphoid cell (ILC2) development and regulation of interleukin-4 (IL-4) and IL-13 production," Cytokine, vol. 75, no. 1, pp. 14-24, 2015.

[21] H. D. Dawson, G. Collins, R. Pyle et al., "Direct and indirect effects of retinoic acid on human Th2 cytokine and chemokine expression by human T lymphocytes," BMC Immunology, vol. 7, article 27, 2006.

[22] E. Kolaczkowska, B. Arnold, and G. Opdenakker, "Gelatinase B/MMP-9 as an inflammatory marker enzyme in mouse zymosan peritonitis: Comparison of phase-specific and cell-specific production by mast cells, macrophages and neutrophils," Immunobiology, vol. 213, no. 2, pp. 109-124, 2008.

[23] E. Kolaczkowska, W. Grzybek, N. van Rooijen et al., "Neutrophil elastase activity compensates for a genetic lack of matrix metalloproteinase-9 (MMP-9) in leukocyte infiltration in a model of experimental peritonitis," Journal of Leukocyte Biology, vol. 85, no. 3, pp. 374-381, 2009.

[24] C. Liang, L. Yang, and S. Guo, "All-trans retinoic acid inhibits migration, invasion and proliferation, and promotes apoptosis in glioma cells in vitro," Oncology Letters, vol. 9, no. 6, pp. 28332838, 2015.

[25] Y. Liu, K. Kimura, T. Orita, S. Teranishi, K. Suzuki, and K.H. Sonoda, "All-trans-retinoic acid inhibition of transforming growth factor- $\beta$-induced collagen gel contraction mediated by human Tenon fibroblasts: role of matrix metalloproteinases," British Journal of Ophthalmology, vol. 99, no. 4, pp. 561-565, 2015.

[26] M. Narvekar, H. Y. Xue, and H. L. Wong, "A novel hybrid delivery system: Polymer-oil nanostructured carrier for controlled delivery of highly lipophilic drug all-trans-retinoic acid (ATRA)," International Journal of Pharmaceutics, vol. 436, no. 1-2, pp. 721-731, 2012.

[27] T.-T. Li, S. Ogino, and Z. R. Qian, “Toll-like receptor signaling in colorectal cancer: carcinogenesis to cancer therapy," World
Journal of Gastroenterology, vol. 20, no. 47, pp. 17699-17708, 2014.

[28] S. Karumuthil-Melethil, M. H. Sofi, R. Gudi, B. M. Johnson, N. Perez, and C. Vasu, "TLR2- and dectin 1-associated innate immune response modulates T-cell response to pancreatic $\beta$ cell antigen and prevents type 1 diabetes," Diabetes, vol. 64, no. 4, pp. 1341-1357, 2015.

[29] C. R. Brandt, "Peptide therapeutics for treating ocular surface infections," Journal of Ocular Pharmacology and Therapeutics, vol. 30, no. 9, pp. 691-699, 2014.

[30] H. Almeida, P. Lobão, C. Frigerio et al., "New thermoresponsiveeyedrop formulation containing ibuprofen loaded-Nanostructured Lipid Carriers (NLC): development, characterisation and biocompatibility studies," Current Drug Delivery, 2015.

[31] J. Zhou and D. Zhou, "Improvement of oral bioavailability of lovastatin by using nanostructured lipid carriers," Drug Design, Development and Therapy, vol. 9, pp. 5269-5275, 2015.

[32] A. Chinsriwongkul, P. Chareanputtakhun, T. Ngawhirunpat et al., "Nanostructured lipid carriers (NLC) for parenteral delivery of an anticancer drug," AAPS PharmSciTech, vol. 13, no. 1, pp. 150-158, 2012. 


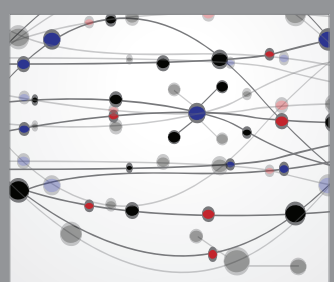

The Scientific World Journal
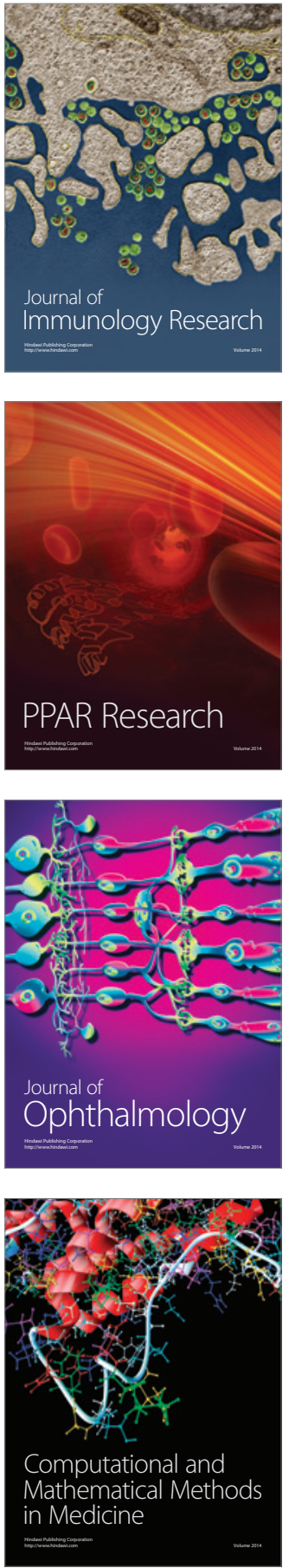

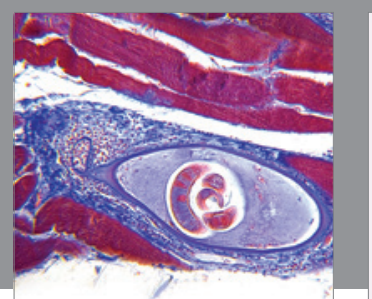

Gastroenterology Research and Practice

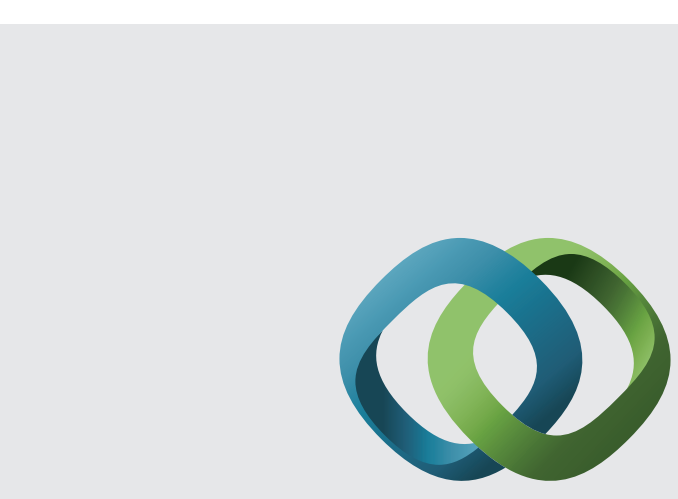

\section{Hindawi}

Submit your manuscripts at

http://www.hindawi.com
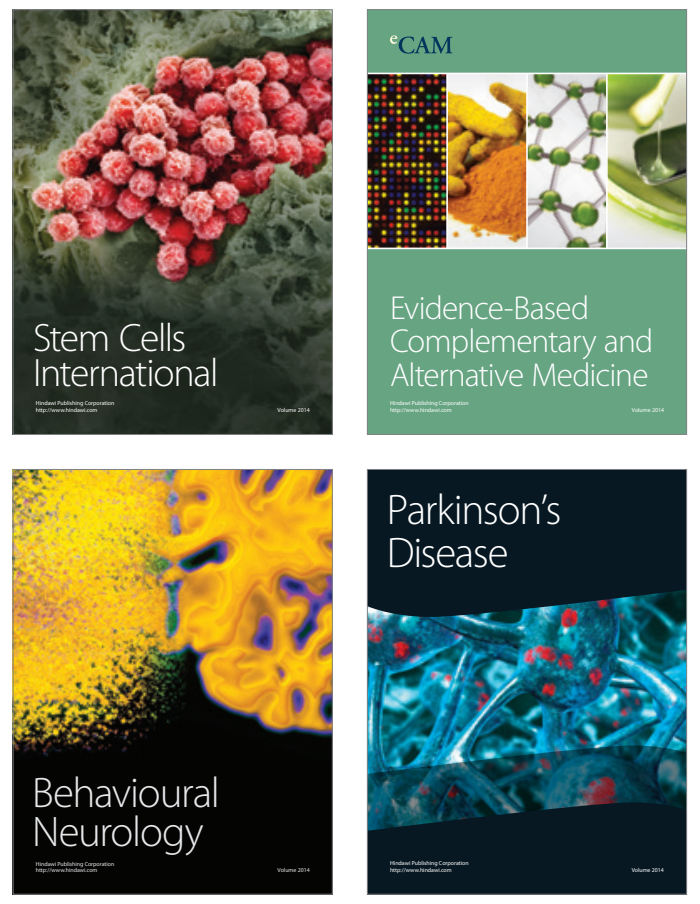
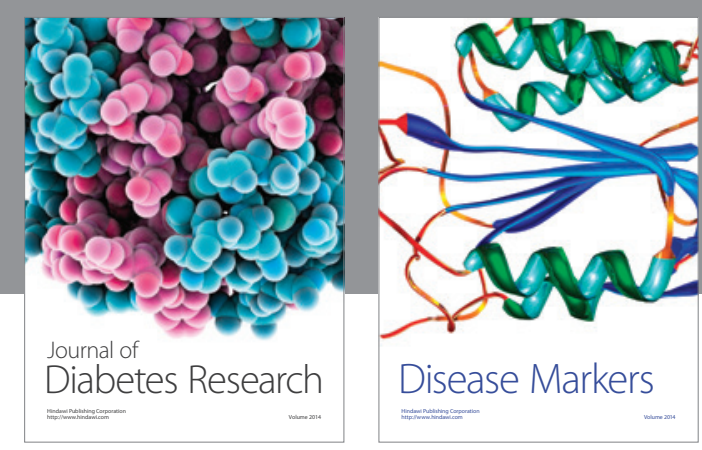

Disease Markers
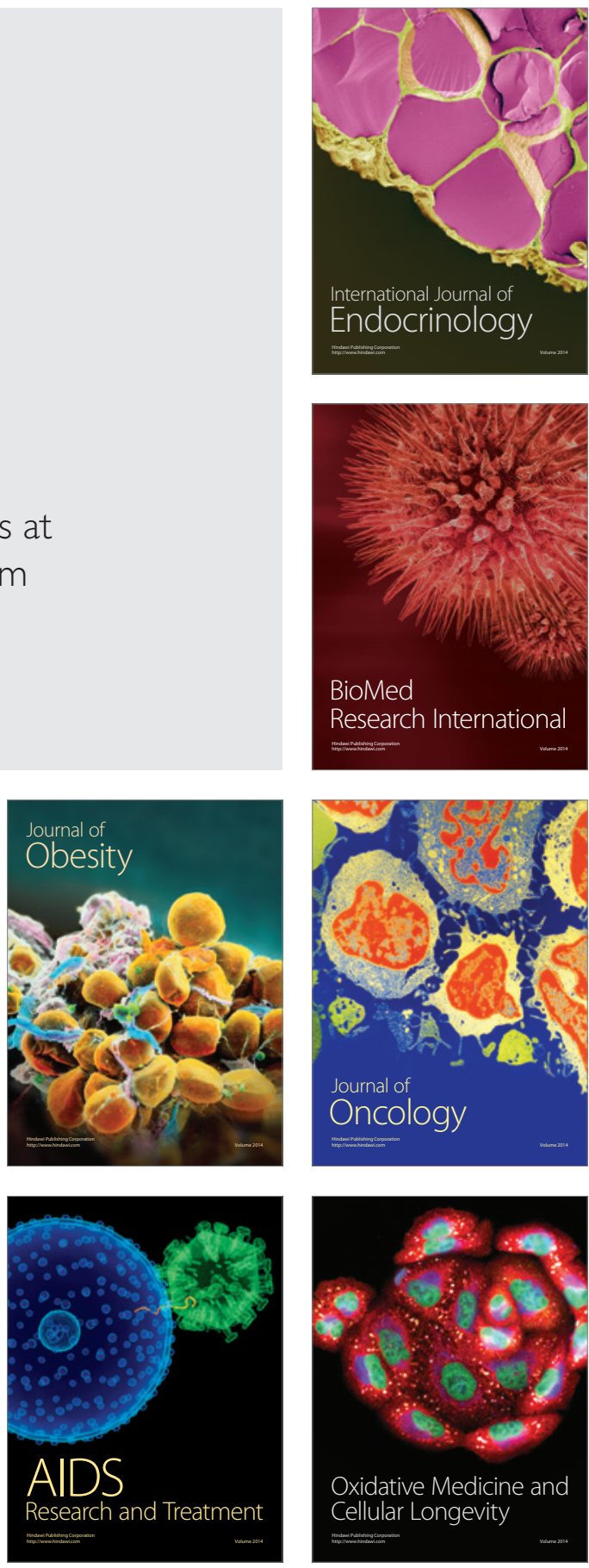